\title{
COMPUTATION OF FLUX INTO THE ALADDIN INFRARED BEAMLINE
}

\author{
R. A. Bosch, Synchrotron Radiation Center, \\ University of Wisconsin-Madison, 3731 Schneider Dr., Stoughton, WI 53589
}

\section{Abstract}

The Aladdin infrared beamline extracts edge radiation downstream of a short straight section of the $800 \mathrm{MeV}$ electron storage ring, Aladdin. For wavelengths of 11000 microns, the flux into the beamline is computed. The flux rolls off at wavelengths exceeding $\sim 100 \mu \mathrm{m}$, where the central cone of near-field edge radiation is no longer contained within the entrance aperture.

\section{INTRODUCTION}

The radiation from electrons entering or exiting a bending magnet (edge radiation [1,2,3,4]) may be used as a bright infrared (IR) source. Recently, an infrared beamline using edge radiation has been constructed downstream of a short straight section of the $800 \mathrm{MeV}$ electron storage ring, Aladdin [5].

The bending magnet fringe field is approximately a linear ramp over a distance of $10.8 \mathrm{~cm}$, while the uniform field region bends the electron orbit with a $2.083 \mathrm{~m}$ radius of curvature. An electron entering the magnet is bent through an angle of $1 / \gamma$ in a distance $d$ of $1.7 \mathrm{~cm}$, where $\gamma$ is the relativistic mass factor. Intense edge radiation may thus be expected for wavelengths $\lambda \gg d / \gamma^{2}=7 \mathrm{~nm}$ [6].

The straight section length $L$ is $3 \mathrm{~m}$, while the entrance aperture is at a distance $R$ of $1.5 \mathrm{~m}$ from the downstream end of the straight section with dimensions of $30.5 \mathrm{~mm}$ horizontal x $22.3 \mathrm{~mm}$ vertical; its full angular extent is 20 mrad x 15 mrad. Near-field broadening is expected to diminish the flux through an aperture for $\lambda>$ $\theta_{1 / 2}{ }^{2} R L /(R+L)$, where $\theta_{1 / 2}$ is the half-angle subtended by the aperture $[7,8]$. The horizontal aperture is expected to cut off flux for $\lambda>(10 \mathrm{mrad})^{2}(1.5 \mathrm{~m})(3 \mathrm{~m}) /(1.5 \mathrm{~m}+3 \mathrm{~m})=$ $100 \mu \mathrm{m}$, while the vertical aperture may cut off flux for $\lambda$ $>(7.5 \mathrm{mrad})^{2}(1.5 \mathrm{~m})(3 \mathrm{~m}) /(1.5 \mathrm{~m}+3 \mathrm{~m})=56 \mu \mathrm{m}$. Intense flux may therefore be expected for $7 \mathrm{~nm} \ll \lambda<<$ 56-100 $\mu \mathrm{m}$.

\section{FLUX COMPUTATION}

The electric field from an electron, in SI units, obeys [9]

$$
\begin{aligned}
\vec{E}(\vec{X}, \omega) & =\frac{e}{4 \pi \varepsilon_{0} c} \int_{-\infty}^{\infty} \frac{\hat{n} \times[(\hat{n}-\vec{\beta}) \times \vec{\beta}]}{(1-\hat{n} \cdot \vec{\beta})^{2} R(t)} e^{i \omega t_{o}(t)} d t \\
& +\frac{e}{4 \pi \varepsilon_{0} \gamma^{2}} \int_{-\infty}^{\infty} \frac{\hat{n}-\vec{\beta}}{(1-\hat{n} \cdot \vec{\beta})^{2} R(t)^{2}} e^{i \omega t_{o}(t)} d t .
\end{aligned}
$$

The first term on the RHS is the "acceleration field" describing far-field radiation. The second term is the "velocity field" which contributes in the near field. Here,
$\vec{X}=(X, Y, Z)$ is the observer location, $\omega$ is angular frequency, $c$ is the speed of light, $\vec{\beta}(t)$ is the electron velocity divided by $c, e$ is the electron charge, and $\varepsilon_{\mathrm{o}}$ is the permittivity of free space. $R(t)$ is the distance from the electron to the observer and $\hat{n}(t)$ is the unit vector pointing from the electron to the observer. The observer time $t_{o}$ is related to the time $t$ of radiation emission by

$$
\frac{d t_{o}}{d t}=1-\hat{n}(t) \cdot \vec{\beta}(t) \text {. }
$$

Consider an electron which enters the fringe field of a bending magnet, located at the coordinate origin, at time $t=0$. Let $R=|\vec{X}|$ and $\hat{n}(0)=(x, y, z)$ describe the distance and direction to the observer from the magnet entrance. Modeling the fringe field as a linear ramp over a distance $l$ to the value $B_{\mathrm{y}}$ in the central region of the magnet, the normalized acceleration, $\dot{\beta} / \beta$, obeys

$$
a(t)=\left\{\begin{array}{cc}
\left(\omega_{c} / \tau\right) t & 0<t<\tau \\
\omega_{c} & t>\tau
\end{array}\right.
$$

where $\omega_{\mathrm{c}}=e B_{\mathrm{v}} / m \gamma$ is negative for a bend in the $+x$ direction, and $\tau \equiv l / \beta c$ is the fringe-field traversal time. Letting $A(t) \equiv \int_{0}^{t} a\left(t^{\prime}\right) d t^{\prime}$, we have

$$
\begin{aligned}
& \vec{\beta}(t)=\beta(-\sin A(t), 0, \cos A(t)), \\
& \overrightarrow{\dot{\beta}}(t)=\beta a(t)(-\cos A(t), 0,-\sin A(t)),
\end{aligned}
$$

and

$$
A(t)= \begin{cases}\left(\omega_{c} t^{2} / 2 \tau\right) & 0<t<\tau \\ \omega_{c}(t-\tau / 2) & t>\tau .\end{cases}
$$

Approximating $\sin A(t) \approx A(t)$ to evaluate the electron position $\left(X_{e}, Y_{e}, Z_{e}\right)$ versus time gives

$$
X_{e}(t)=\left\{\begin{array}{lr}
-\frac{\beta c \omega_{c} t^{3}}{6 \tau} & 0<t<\tau \\
-\beta c \omega_{c}\left[\frac{\tau^{2}}{24}+\frac{(t-\tau / 2)^{2}}{2}\right] & t>\tau
\end{array}\right.
$$

and $Z_{e}(t) \approx \beta c t$.

From eq. (2) we have, for $t_{o}(0) \equiv 0$

$$
\begin{aligned}
t_{o}(t)= & \int_{0}^{t}\left[1-\hat{n}(0) \cdot \vec{\beta}\left(t^{\prime}\right) d t^{\prime}\right]+\int_{0}^{t}\left[\hat{n}(0)-\hat{n}\left(t^{\prime}\right)\right] \cdot \vec{\beta}(0) d t^{\prime} \\
& +\int_{0}^{t}\left[\hat{n}(0)-\hat{n}\left(t^{\prime}\right)\right] \cdot\left[\vec{\beta}\left(t^{\prime}\right)-\vec{\beta}(0)\right] d t^{\prime}
\end{aligned}
$$


The first term on the RHS of eq. (8) is the expression for $t_{o}(t)$ applicable when the time-variation of $\hat{n}(t)$ may be neglected, i.e. for a distant observer. Its value is [2]

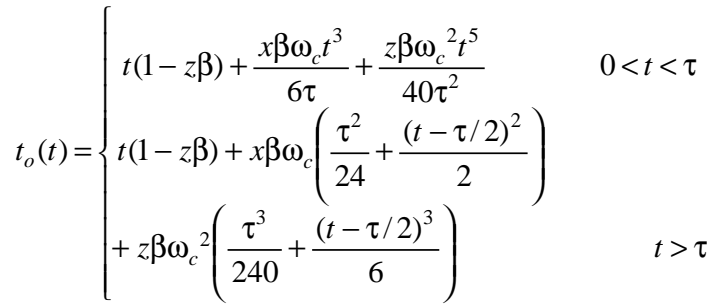

The second term on the RHS of eq. (8) is approximately

$$
\begin{aligned}
& \Delta t_{o}(t)=\left(x^{2}+y^{2}\right)\left(\frac{\beta^{2} c t^{2}}{2 R}+\frac{\beta^{3} c^{2} t^{3}}{3 R^{2}}\right)+\frac{\beta^{2} c \omega_{c} x}{R}\left(\frac{t^{4}}{24 \tau}+\frac{\beta c t^{5}}{30 R \tau}\right) 0<t<\tau \\
&=\left(x^{2}+y^{2}\right)\left(\frac{\beta^{2} c t^{2}}{2 R}+\frac{\beta^{3} c^{2} t^{3}}{3 R^{2}}\right)+ \\
& \frac{\beta^{2} c \omega_{c} x}{R}\left(\frac{t^{3}}{6}-\frac{t^{2} \tau}{4}+\frac{t \tau^{2}}{6}-\frac{\tau^{3}}{24}+\frac{\beta c}{R}\left(\frac{t^{4}}{8}-\frac{t^{3} \tau}{6}+\frac{t^{2} \tau^{2}}{12}-\frac{\tau^{4}}{120}\right)\right) t>\tau
\end{aligned}
$$

The dominant contribution to the third term is

$$
\delta t_{o}(t)=\left\{\begin{array}{lr}
\frac{\beta^{2} c \omega_{c} x}{R}\left(\frac{t^{4}}{8 \tau}+\frac{\beta c t^{5}}{10 R \tau}\right) & 0<t<\tau \\
\frac{\beta^{2} c \omega_{c} x}{R}\left(\frac{t^{3}}{3}-\frac{t^{2} \tau}{4}+\frac{\tau^{3}}{24}+\frac{\beta c}{R}\left(\frac{t^{4}}{4}-\frac{t^{3} \tau}{6}+\frac{\tau^{4}}{60}\right)\right) & t>\tau
\end{array}\right.
$$

The radiation emitted when $t>0$ may be evaluated by substituting eqs. (3)-(11) into eq. (1) and numerically integrating over $0<t<t_{\max }$, where $t_{\max }$ is sufficiently large that radiation from later times is negligible. By including eqs. (10) and (11), the expression for $t_{o}(t)$ is sufficiently accurate that computations agree with the results of Chubar [8] for observer locations within the Aladdin IR beamline entrance aperture. However, inaccurate results are obtained for the ordinary synchrotron radiation observed at larger horizontal displacements. This is an apparent limitation of the approximate expression for $t_{o}(t)$.

Now consider radiation emitted before this electron enters the straight section at time $t=-L / \beta c$, where $L$ is the straight section length. If we instead consider an electron entering the straight section at time $t=0$, where $t_{o}(0) \equiv 0$, eqs. (3)-(11) apply with the substitutions: $R \rightarrow R+L, \tau \rightarrow-$ $\tau, 0<t<\tau \rightarrow-\tau<t<0, t>\tau \rightarrow t<-\tau$; the unit vector pointing from the upstream straight-section end to the observer must be substituted for $(x, y, z)$. Inserting these expressions into eq. (1), we integrate numerically over $-t_{\max }$ $<t<0$ to obtain $\vec{E}(\vec{X}, \omega)$. To convert $\vec{E}(\vec{X}, \omega)$ to the observer time coordinate where $t_{o}=0$ when downstream edge radiation arrives, we multiply by the phase $\exp \left\{-\mathrm{i} \pi R_{n}(\lambda)\left[L / R+\phi(X, Y)^{2} L /(R+L)\right]\right\}, \quad$ where $R_{n}(\lambda) \equiv$ $R /\left(\lambda \gamma^{2}\right)$ and $\phi(X, Y) \equiv \gamma\left(x^{2}+y^{2}\right)^{1 / 2}$.

Lastly, the velocity field from the straight section is [7]

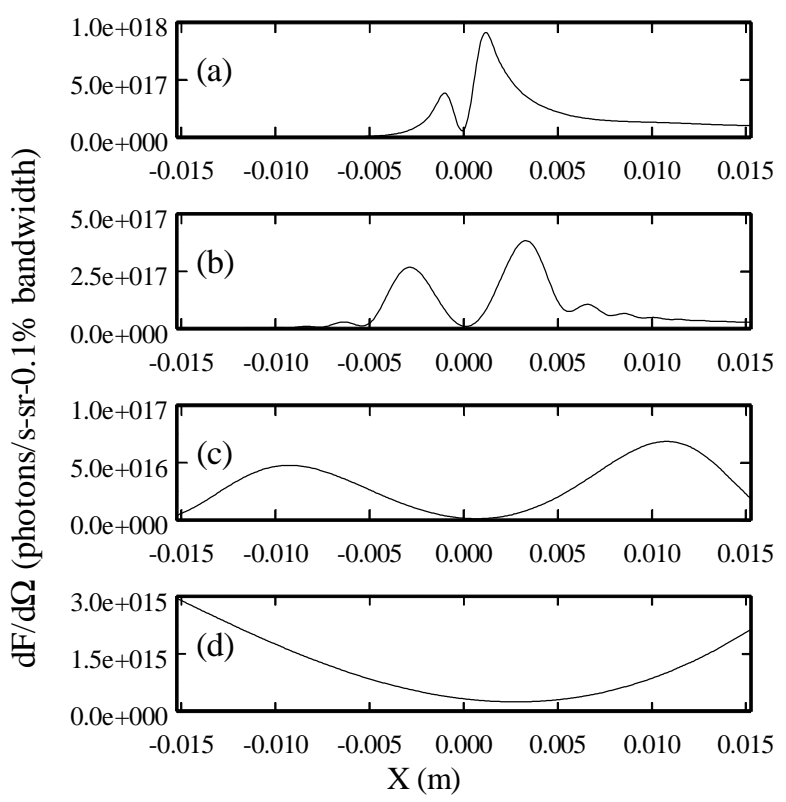

Figure 1. Flux density $1.5 \mathrm{~m}$ downstream of the entrance edge of an Aladdin bending magnet preceded by an infinitely-long straight section, for an electron current of $200 \mathrm{~mA}$ and energy of $800 \mathrm{MeV}$. Flux density is plotted versus horizontal position in the plane of the electron orbit, for electrons deflected in the positive $x$-direction. (a) $\lambda=1 \mu \mathrm{m}$. (b) $\lambda=10 \mu \mathrm{m}$. (c) $\lambda=100 \mu \mathrm{m}$. (d) $\lambda=1000 \mu \mathrm{m}$.

$$
E(\vec{X}, \lambda)=\left(\frac{e \gamma}{\pi \varepsilon_{0} c R}\right)\left(\frac{e^{i \pi R_{n}\left(1-\phi^{2}\right)}}{\phi}\right) \int_{R \phi}^{\phi} \frac{w}{(1+L} \frac{\left.w w^{2}\right)^{2}}{i \pi R_{n} \phi(w-1 / w)} d w
$$

The $x$ - and $y$-components of eq. (12) are obtained by multiplying by $x /\left(x^{2}+y^{2}\right)^{1 / 2}$ and $y /\left(x^{2}+y^{2}\right)^{1 / 2}$.

Summing the electric fields from the edges and the straight section gives the electric field from an electron. For a bunch length exceeding the observed radiation wavelength, the flux density from a current, $I$, is [9]

$$
\frac{d F}{d \Omega}=\alpha \frac{\Delta \omega}{\omega} \frac{I}{e}\left(\frac{2 R \varepsilon_{0} c}{e}\right)^{2}|\vec{E}(\vec{X}, \omega(\lambda))|^{2},
$$

where the solid angle $d \Omega$ is defined with respect to the downstream bending magnet entrance.

\section{RESULTS}

Figure 1 displays the computed flux density $1.5 \mathrm{~m}$ downstream of an Aladdin bending magnet preceded by an infinitely-long straight section. The flux density in the plane of the electron orbit is plotted versus horizontal position $X$ for locations within the IR beamline entrance aperture. For the wavelengths shown, $\lambda$ exceeds $R / \gamma^{2}$, so that near-field effects must be considered [7].

At a wavelength of $1 \mu \mathrm{m}, \lambda$ is slightly larger than $R / \gamma^{2}$ $\left(\lambda=1.75 R / \gamma^{2}\right)$, and the velocity field makes only a small contribution. The peaks of flux density are approximately 


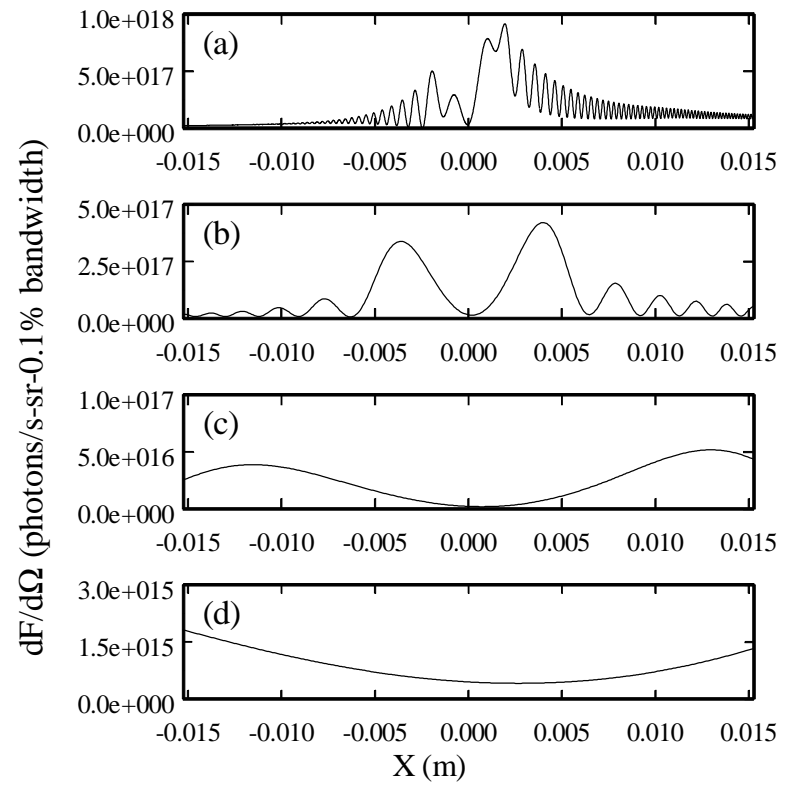

Figure 2. Flux density $1.5 \mathrm{~m}$ downstream of the entrance edge of an Aladdin bending magnet, for a current of $200 \mathrm{~mA}$ and straight section length of $3 \mathrm{~m}$. Flux density is plotted versus horizontal position in the plane of the electron orbit, for electrons deflected in the positive $x$-direction. (a) $\lambda=1 \mu \mathrm{m}$. (b) $\lambda=10 \mu \mathrm{m}$. (c) $\lambda=100 \mu \mathrm{m}$. (d) $\lambda=1000 \mu \mathrm{m}$.

at the far-field angles of $\pm 1 / \gamma= \pm 0.64 \mathrm{mrad}$. For large positive values of $X$, the radiation is similar to ordinary synchrotron radiation. At wavelengths of $10-1000 \mu \mathrm{m}, \lambda$ greatly exceeds $R / \gamma^{2}$, so that the peaks of the flux density occur at $X \approx \pm(\lambda R)^{1 / 2}$ [7]. For wavelengths of $1-100 \mu \mathrm{m}$, the central cone of radiation lies within the horizontal aperture, while most of this cone lies outside the aperture for $\lambda=1000 \mu \mathrm{m}$.

Figure 2 shows the computed flux density for the Aladdin short straight section length $L$ of $3 \mathrm{~m}$. At $\lambda=1$ $\mu \mathrm{m}$, the straight section length exceeds the "radiation formation length" of $\lambda \gamma^{2}$. The interference between the two ends of the straight section results in fringe spacing small compared to the angular scale of the single-edge flux distribution. At the longer wavelengths of 10-1000 $\mu \mathrm{m}$, where $\lambda$ greatly exceeds $R / \gamma^{2}$ and $L / \gamma^{2}$, the peaks of the flux density occur at $X \approx \pm(\lambda R(R+L) / L)^{1 / 2}$ [8]. The peaks are $\sim 20 \%$ further from the straight-section axis than with an infinite straight section length. Again, the central radiation cone is largely contained within the horizontal aperture for wavelengths of $1-100 \mu \mathrm{m}$.

Figure 3 shows computed flux into the IR beamline for the Aladdin short straight section length of $3 \mathrm{~m}$ and also for an infinite straight section length. For $\lambda>100 \mu \mathrm{m}$, the flux is diminished because the central cone of near-field radiation is not contained within the entrance aperture, consistent with our estimate that the aperture will reduce flux for $\lambda>56-100 \mu \mathrm{m}$. We also show the flux into an

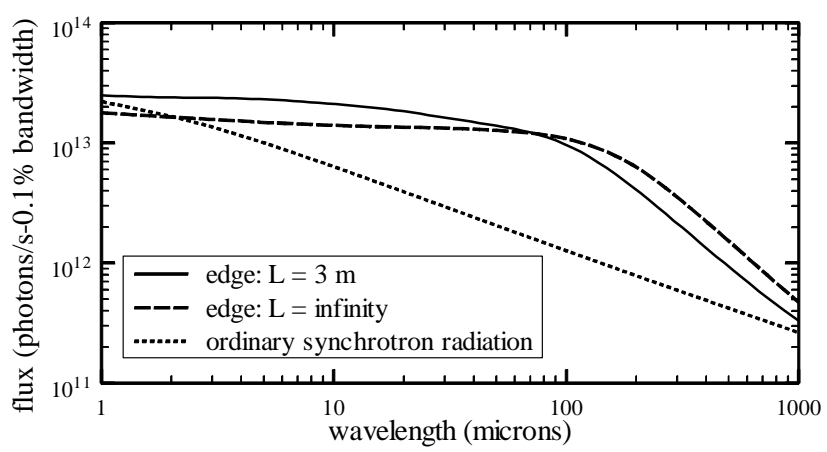

Figure 3. Computed flux into the Aladdin IR beamline for an electron current of $200 \mathrm{~mA}$ (solid line). The edge-radiation flux for an infinite straight section length and the ordinary synchrotron radiation flux into an identical aperture are also shown.

identical aperture located $1.5 \mathrm{~m}$ downstream of an ordinary synchrotron radiation source at Aladdin.

\section{SUMMARY}

The flux of edge radiation into the Aladdin IR beamline has been computed. The computations show a large flux at the wavelengths of 1-20 $\mu \mathrm{m}$ typically used for Fourier transform infrared (FTIR) microscopy. At wavelengths exceeding $\sim 100 \mu \mathrm{m}$, the flux is diminished.

\section{ACKNOWLEDGMENTS}

The author appreciates discussions with O. V. Chubar, M. A. Green, R. L. Julian, T. E. May, R. Reininger and W. S. Trzeciak. This work was supported by NSF grant DMR95-31009.

\section{REFERENCES}

[1] O. V. Chubar and N. V. Smolyakov, J. Optics (Paris) 24, 117 (1993); Proc. 1993 IEEE PAC, 1626 (1993).

[2] R. A. Bosch, T. E. May, R. Reininger and M. A. Green, Proc. SRI '95 (CD ROM), Rev. Sci. Instrum. 67, 3346(K) (1996).

[3] R. A. Bosch, Nucl. Instr. and Meth. A386, 525 (1997).

[4] Y.-L. Mathis, P. Roy, B. Tremblay, A. Nucara, S. Lupi, P. Calvani and A. Gerschel, Phys. Rev. Lett. 80, 1220 (1998).

[5] T. E. May, R. A. Bosch and R. L. Julian, "Infrared edge radiation beamline at Aladdin," these proceedings.

[6] R. A. Bosch, Il Nuovo Cimento D20, 483 (1998).

[7] R. A. Bosch and O. V. Chubar, Proc. SRI '97, Tenth US National Conference, AIP Conf. Proc. 417 (AIP, NY, 1997) p. 35.

[8] O. V. Chubar, "Presentation of a computer code and results on a common problem," in Workshop on Infrared Synchrotron Radiation, 1st Meeting: The Source, organized by J.-L. Laclare, M.-P. Level and P. Roy (1997).

[9] J. D. Jackson, Classical Electrodynamics, 2nd ed. (Wiley, NY, 1975) p. 670. 